\title{
Propolis as an Anti-allergy Based on Decrease in Total Eosinophil Count in Rat Models
}

\author{
Rashmika Nambiar, ${ }^{1}$ Enny Rohmawaty, ${ }^{2}$ Leni Lismayanti ${ }^{3}$ \\ ${ }^{1}$ Faculty of Medicine Universitas Padjadjaran, ${ }^{2}$ Department of Pharmacology and Therapy, \\ Faculty of Medicine, Universitas Padjadjaran, ${ }^{3}$ Department of Clinical Pathology Faculty of \\ Medicine, Universitas Padjadjaran/Dr. Hasan Sadikin General Hospital Bandung
}

\begin{abstract}
Background: Propolis is a natural herb derived from plant resins by bees. Propolis contains flavonoids that act as anti-allergy. The composition of flavonoids in propolis varies according to each region like Brazilian, Chinese, Malaysian and Indonesian propolis due to differences in medicinal plants in that area. The aim of this study is to determine the effect of propolis as an anti-allergy and to determine which is the most effective among propolis of Brazilian, Malaysian and Indonesian origin.

Methods: An experimental study was performed on 28 Wistar male rats divided into four groups, Brazilian, Malaysian, Indonesian propolis and a negative control group. The used parameter was decreased in total eosinophil count in ovalbumin induced allergy in rats. Propolis or aquadest as control were given orally $0.25 \mathrm{ml}$ once daily.

Results: All three groups of propolis showed statistically significantresults $(\mathrm{p}<0.05)$, in decreasing eosinophil count. However, Malaysian and Brazilian propolis showed much more significant effects compared to that of Indonesian propolis. This could be due to the difference in composition and concentration of flavonoids in Indonesian propolis compared to the Brazilian and Malaysian propolis.

Conclusion:Propolis has a significant effect as an anti-allergy. Malaysian and Brazilian propolis are more effective as an anti-allergy compare to Indonesian propolis. Propolis can thus be used as an alternative treatment for allergy. [AMJ.2015;2(1):208-12]
\end{abstract}

Keywords: Anti-allergy, eosinophil count, propolis, rats

\section{Introduction}

Allergy is a hypersensitivity reaction initiated by the immune response of our body and Indonesia is classified as one of the countries with low allergy prevalence prompting the researcher to think that probably traditional medicine is the reason behind this data. ${ }^{1}$ Egg allergy is very common and known to contain ovalbumin due to many peptides that can trigger an allergic reaction. Thus, it spurs the researcher to use ovalbumin to induce allergy for research. ${ }^{2}$ Allergy causes itch, pain, and swollen areas (edema). The definite diagnosis of allergies are increased in total eosinophil count and IgE level in blood. ${ }^{3}$ The currently available treatments for allergy are antihistamines and corticosteroids. Both having side effects, but still used, due to the necessity and demand in society. ${ }^{4}$

Natural herbs have been gaining popularity in the discovery of new alternative treatment recently due to scarce side effects and its common used in the society. Indonesia is known to contain almost 30,000 herbs. In the last decades, propolis emerges as a natural herb rigorously researched to determine the treatment of allergy using propolis. ${ }^{5}$ Propolis is derived from plant resins by bees and can act as anti-microbial, anti-inflammatory, and immunostimulatory. ${ }^{6}$ Researchers have claimed that propolis containing similar active components worldwide but the composition is actually various depending on the area in which they are collected due to variation in wild plants in that area. ${ }^{7}$ There are many types of propolis, like Brazilian, Chinese, Malaysian and Indonesian propolis. ${ }^{8}$

According to a research done on Chinese and Brazilian propolis, the possible antiallergic components identified are flavonoids, which are kaempherol and chrysin., ${ }^{7,9}$ In another study, caffeic acid phenyl ester (CAPE) was also considered as possible antiallergic component. ${ }^{10,11}$ Malaysia and Brazilian

Correspondence: Rashmika Nambiar, Faculty of Medicine, Universitas Padjadjaran, Jalan Raya Bandung-Sumedang Km.21, Jatinangor, Sumedang, Indonesia, Phone: +628170205818 Email: ngiaren31mika@hotmail.com 
propolis both contain flavonoids prompting the researcher to use these propolis and also Indonesian propolis, in which there are fewer publications regarding its components and composition to determine the use of propolis as an anti-allergic.

The aim of this research is to study the effect of propolis as an anti-allergy based on decrease in total eosinophil count in ovalbumin-induced allergy in rats and to determine which is the most effective among propolis of Brazilian, Malaysian and Indonesian origin as an antiallergy that will induce further researches to determine the safety of propolis in the field of phytopharmaca and establish propolis as an easy obtainable anti-allergic drug in the future.

\section{Methods}

An experimental study was done to investigate the effects of propolis as an anti-allergy in ovalbumin-induced allergy in rats. Twenty eight adult Wistar male rats were divided randomly into 4 groups containing 7 rats each. The experimental rats were obtained from Bandung Institute of Technology and underwent an adaptation period for 7 days at Pharmacology lab, Faculty of Medicine, Universitas Padjadjaran, Bandung. They were fed pellets and drank distilled water.

A preliminary test was done before the main research was conducted. The aim was to confirm whether ovalbumin 2\% (SigmaAldrich) can induce allergy in rats. After 1 week adaptation, the total eosinophil count of 5 healthy rats was obtained. Four rats were induced with ovalbumin $2 \% 3$ times while 1 rat acted as control. Total eosinophil count was obtained again and the rise in total eosinophil count compared to baseline indicated an allergic reaction. The control did not show a significant increase in eosinophil count concluding that ovalbumin $2 \%$ was sufficient to induce an allergic reaction in rats.

The main experimental research started when the rats were adapted for 7 days and the total eosinophil count was obtained again as a baseline. The rats of groups A, B, C and D were then further induced by $0.5 \mathrm{ml}$ ovalbumin $2 \%$ subcutaneously on day 0,7 , and 14 . On day 16 , the total eosinophil count was obtained again for all the four groups.

After obtaining blood sample for total eosinophil count on day 16, the adult Wistar male rats in the four groups continued once daily oral administration $(0.25 \mathrm{ml})$ of propolis Indonesia-Trigona for group A, propolis Malaysia-Melia for group B, propolis BrazilMinas Gerais for group $\mathrm{C}$ and aquadest for group $\mathrm{D}$ for a duration of 7 days. On day 23, the total eosinophil count was determined again. The total eosinophil count on day 16 and day 23 were further used as data for statistical analysis.

Total eosinophil count in blood were determined using the direct method of absolute eosinophil count which was the principle of hemocytometry and this used improved Neubauer counting chamber and Dunger's solution. Dunger's solution comprised of aqueous eosin $1 \%$, acetone $10 \mathrm{ml}$ and distilled water $100 \mathrm{ml}$. Blood was diluted 10 times in a WBC pipette with Dunger's solution which removed red cells and stained eosinophils. The diluted blood specimen was then placed in a counting chamber and the cells were counted under a high power objective lens of a microscope. Eosinophil granules stained orange-red and other WBC nuclei stained bluegreen. Eosinophils were then calculated for the undiluted blood. Cells in $1 \mathrm{~mm} 3$ of undiluted

Table 1 Mean Decrease in Total Eosinophil Count of Rats (cell count/ $\mu \mathrm{l}$ )

\begin{tabular}{|c|c|c|c|c|c|}
\hline No & Group A & Group B & Group C & Group D & $\mathbf{p}$ \\
\hline 1 & 159 & 134 & 145 & 67 & \\
\hline 2 & 89 & 88 & 66 & 122 & \\
\hline 3 & 122 & 156 & 134 & 0 & \\
\hline 4 & 123 & 178 & 167 & 67 & \\
\hline 5 & 89 & 167 & 88 & 22 & \\
\hline 6 & 111 & 111 & 134 & 67 & \\
\hline 7 & 56 & 133 & 178 & 33 & \\
\hline Mean \pm SD & $107,00 \pm 32,79$ & $138,14 \pm 31,71$ & $130,29 \pm 40,39$ & $54,00 \pm 39,82$ & 0.001 \\
\hline
\end{tabular}

$\mathrm{SD}=$ Standard Deviation 
blood $=(10 \times 10) / 9 \times 7$. Dunger's solution comprised of aqueous eosin $1 \%$, acetone 10 $\mathrm{mL}$ and distilled water $100 \mathrm{~mL} .12$

All the data from Group A,B, C and D were statistically analyzed using one way Analysis of Variance (ANOVA) by SPSS 15.0 software, to compare differences between the four independent groups. The $\mathrm{p}$ value $<0.05$ was considered as significant.

\section{Results}

Based on the preliminary study, the 4 rats induced with ovalbumin $2 \%$ showed a significant increase in eosinophil count compare to the baseline.

The main research was divided into two. First, to determine the effects of propolis from Brazil, Indonesia and Malaysia compared to aquadest to test whether these propolis had anti-allergic effects and second, to determine which propolis showed most effective antiallergic effect. The data collected were analyzed for the decrease in total eosinophil count.

All groups showed differences in total eosinophil count compared to baseline. The largest difference in mean decrease showed by group B (Malaysia) and followed closely by group C (Brazil).

The effect of propolis Indonesia (Trigona), Malaysia (Melia) and Brazil (Minas Gerais) as anti-allergic compared to aquadest was further tested by using one way Analysis of
Variance (ANOVA).

The ANOVA test produced a result $p$-value $<0.05$, indicating there was a significant difference between groups, thus, proving all three groups of propolis acts as anti-allergic. The best anti-allergic effect among propolis Indonesia (Trigona), Malaysia (Melia) and Brazil was done by post hoc Tukey test.

Groups B and C clearly showed statistically significant effect of propolis as an anti-allergic because the $p$-value was $<0.05$. If the standard error used was $10 \%$ instead of $5 \%$, then group A- Indonesia would also be statistically significant. As a conclusion, propolis from Malaysia, Brazil and Indonesia had statistically significant effects in acting as an anti-allergic, confirmed by a decrease in eosinophil count. (Table 2)

\section{Discussions}

Ovalbuminwasinjected 3 timessubcutaneously to ensure optimal sensitization for a healthy rat to have an allergic reaction. All propolis groups, Indonesia, Malaysia and Brazilian propolis acted as anti-allergy, but in this study, Malaysian and Brazilian propolis, suggested that they were statistically much more significant as an anti-allergy compare to Indonesian propolis. Since active compounds in all propolis are the same, the difference in composition and concentration of propolis plays the role in explaining the difference in significance.

Table 2 Effectiveness of each propolis

\begin{tabular}{llc}
\hline \multicolumn{1}{c}{ Source } & \multicolumn{1}{c}{ Comparison Groups } & p \\
\hline Group A (Trigona) & Group B (Melia) & 0.397 \\
& Group C (Brazil) & 0.635 \\
Group B (Melia) & Group D (Aquadest) & 0.054 \\
& Group A (Trigona) & 0.397 \\
& Group C (Brazil) & 0.977 \\
Group C (Brazil) & Group D (Aquadest) & $0.001^{*}$ \\
& Group A (Trigona) & 0.635 \\
Group D (Aquadest) & Group B (Melia) & 0.977 \\
& Group D (Aquadest) & $0.003^{*}$ \\
& Group A (Trigona) & 0.054 \\
\hline
\end{tabular}

$* \mathrm{p}<0.05$ 
The most important active components which plays a role as an anti-allergy are flavonoids, which are kaempherol and chrysin. ${ }^{13}$ Both Brazilian and Malaysian propolis contain flavonoids. And in Indonesian propolis, the active compounds are yet to be determined as there are fewer publications regarding Indonesian propolis. However, they might have fewer amounts of flavonoids in $1 \mathrm{~mL}$ of Indonesian propolis or perhaps the concentration or dilution of Indonesian propolis is various compared to other propolis making it statistically less significant.

Chrysin and Kaempherol act as antioxidant, whereby they stabilize the reactive oxygen species, preventing tissue damage in allergy. ${ }^{14,15}$ Chrysin inhibits IL-4 and decreases gene expression of pro-inflammatory cytokine expression preventing eosinophil activation causing decrease in total eosinophil count observed in this research. ${ }^{7}$ Kaempherol also inhibits MAPK (protein kinases) activation in IL-4, decreasing proliferation of IL-4, thereby decreasing eosinophil activation as above. ${ }^{16}$ Furthermore, kaempherol suppresses CD23 mRNA expression preventing second signal for mast cell degranulation to occur, and thus cytokine release decreases indirectly causing eosinophil count to decrease. ${ }^{7,17}$ As a summary, flavonoids like kaempherol and chrysin causes a decrease in eosinophil count as observed in this research.

Compared to other researches, a study conducted in Japan, proved Brazilian and Chinese propolis had effects on mast cell degranulation and cytokine production explaining the decrease in eosinophil count in this case. ${ }^{7}$ Another research in Cihna exhibited an immunoregulatory activity in an ovalbumin sensitized airway inflammatory animal model thus indicating decrease in cytokine production leads to decrease in eosinophil count in this study. ${ }^{18}$

To conclude, propolis has an anti-allergic effect on ovalbumin induced allergy in rats based on the decrease in total eosinophil count. Propolis from Malaysia and Brazil are more effective than propolis Indonesia in acting as anti-allergy. Thus, propolis can be used as an alternative medicine for anti-allergy.

However, it would be better to determine the optimal dosage of propolis Indonesia, Malaysia and Brazil based on toxicity test to determine the safe dosage to be used in the community. There should be further clinical investigations performed on humans so that propolis as antiallergy can be used as an alternative therapy in society. Propolis as an anti-allergy should be promoted to the community so that the society can understand the benefits of propolis as an alternative to the modern drugs.

\section{References}

1. Yazdanbakhsh M, Wahyuni S. The role of helminth infections in protection from atopic disorders. Curr Opin Allergy Clin Immunol 2005; 5(5):386-91

2. Huntington JA, Stein PE. Structure and properties of ovalbumin. J Chromatogr B Biomed Sci Appl. 2001;756(1-2):189-98.

3. Oppenheimer J, Nelson HS. Skin testing. Ann Allergy Asthma Immunol. 2006;96(2 Suppl 1):S6-12.

4. Simons FE. Advances in H1-antihistamines. N Eng J Med. 2004;351(21):2203-17.

5. Sforcin JM, Bankova V. Propolis: is there a potential for the development of new drugs?. J Ethnopharmacol. 2011;133(2):253-60.

6. Khalil ML. Biological activity of bee propolis in health and disease. Asian Pac J Cancer Prev. 2006;7(1):22-31.

7. Nakamura R, Watanabe K, Oka K, Ohta S, Mishima S, Teshima R. Effects of propolis from different areas on mast cell degranulation and identification of the effective components in propolis. Int Immunopharmacol. 2010;10(9):1107-12.

8. Simone-Finstrom M, Spivak M. Propolis and bee health: the natural history and significance of resin use by honey bees. Apidologie. 2010;41(3):295-311.

9. Nijveldt RJ, Van Nood E, van Hoorn DE, Boelens PG, van Norren $K$, van Leeuwen PA. Flavonoids: a review of probable mechanisms of action and potential applications. Am J Clin Nutr. 2001;74(4):418-25.

10. Park SG, Lee DY, Seo SK, Lee SW, Kim SK, Jung WK, et al. Evaluation of anti-allergic properties of caffeic acid phenethyl ester in a murine model of systemic anaphylaxis. Toxicol Appl Pharmacol. 2008;226(1):229.

11. Jung WK, Lee DY, Choi YH, Yea SS, Choi I, Park SG, et.al. Caffeic acid phenethyl ester attenuates allergic airway inflammation and hyperresponsiveness in murine model of ovalbumin-induced asthma. Life Sci. 2008;82(13-14):797-805.

12. Pagana KD, Pagana TJ. Mosby's Diagnostic and Laboratory Test Reference.. 10th ed. St. Louis: Elsevier Mosby; 2010.

13. Bankova V. Recent trends and important 
developments in propolis research. Evid Based Complement Alternat Med. 2005;2(1):29-32.

14. Hamid F, Wiria AE, Wammes LJ, Kaisar MMM, Lell B, Ariawan I, et al. A longitudinal study of allergy and intestinal helminth infections in semi urban and rural areas of Flores, Indonesia (ImmunoSPIN Study). BMC Infect Dis. 2011;11:83.

15. Nakajima Y, Tsuruma K, Shimazawa M, Mishima S, Hara H. Comparison of bee products based on assays of antioxidant capacities. BMC Complement Altern Med. 2009;9:4.

16. Hirose E, Matsushima M, Takagi K, Ota Y,
Ishigami K, Hirayama T, et al. Involvement of heme oxygenase-1 in kaempferolinduced anti-allergic actions in RBL-2H3 cells. Inflammation. 2009;32(2):99-108.

17. Bae Y, Lee S, Kim SH.Chrysin suppresses mast cell-mediated allergic inflammation: Involvement of calcium, caspase- 1 and nuclear factor- $\mathrm{\kappa B}$. Toxicol Appl Pharmacol. 2011;254(1):56-64.

18. Sy LB, Wu YL, Chiang BL, Wang YH, $\mathrm{Wu}$ WM. Propolis extracts exhibit an immunoregulatory activity in an OVAsensitized airway inflammatory animal model. Int Immunopharmacol. 2006;6(7): 1053-60. 